\title{
Integrating III-V quantum dot lasers on silicon substrates for silicon photonics
}

\author{
M. Liao \\ S. Chen \\ M. Tang \\ J. Wu \\ W. Li \\ K. Kennedy \\ I. Ross \\ A. Seeds \\ H. Liu
}




\title{
Integrating III-V quantum dot lasers on silicon substrates for silicon photonics
}

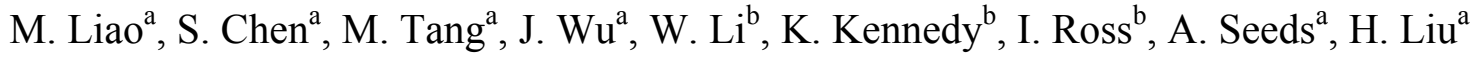 \\ ${ }^{\mathrm{a}}$ Department of Electronic and Electrical Engineering, University College London, London WC1E 7JE, UK \\ ${ }^{b}$ Department of Electronic and Electrical Engineering, University of Sheffield, Sheffield S1 3JD, UK
}

\begin{abstract}
The realization of efficient III-V lasers directly grown on Si substrates is highly desirable for large-scale and low-cost silicon based optoelectronic integrated circuits. However, it has been hindered by the high threading dislocation (TD) density generated at the interface between III-V compounds and Si substrates. Introducing dislocation filter layers (DFLs) to suppress the TD propagation into the active region becomes a key factor for realising lasers with advanced performance. In this paper, optimization of InGaAs/GaAs DFLs in III-V quantum $\operatorname{dot}(\mathrm{QD})$ lasers on Si is demonstrated. Based on these optimized DFLs and other strategies, we have achieved a high performance electrically pumped QD laser on a Si substrate with threshold current density of $62.5 \mathrm{~A} \mathrm{~cm}^{-2}$, over $105 \mathrm{~mW}$ output power, maximum operation temperature of $120^{\circ} \mathrm{C}$ and over $100,158 \mathrm{~h}$ of extrapolated lifetime.
\end{abstract}

Keywords: Molecular beam epitaxy, quantum dots, lasers, silicon photonics

\section{INTRODUCTION}

According to the Moore's law, the number of transistors per square inch on integrated circuit (IC) doubles every 18 months [1], increasing processing speed. However, the shortcomings of metal interconnections increase as the speed increases, such as heat dissipation, current leakage and chemical or material issues. Substituting metal connections with optical connections is one of the promising ways around these limitations. Although $\mathrm{Si}$ photonics has been studied intensively and has borne fruit [1], a reliable and high-efficiency Si-based lightemitting source remains a research challenge due to the indirect bandgap structure of silicon which is not an efficient emitter material [2,3]. Comparing with Si, III-V compounds have superior electronic and optical properties as the active region for a light emitter. Thus, the integration of III-V compounds on Si substrates, by wafer bonding or monolithic growth, has been considered as the most practical approach to achieve a Si-based light emitter [5-12]. Despite the attractive results, with milliwatt output power and high temperature operation (over $100^{\circ} \mathrm{C}$ ) being achieved from lasers by using wafer bonding [13], this method still suffers from low yield for massive production. Monolithic growth of III-V compounds on Si substrates, provides an ideal solution for laser fabrication.

However, there are still some issues that limit the performance of lasers when using monolithic growth method. Firstly, due to the polar and non-polar epitaxial growth of III-V compounds and Si substrate, the formation of antiphase domains (APDs) between III-V/Si results. Moreover, lattice mismatch and incompatible thermal expansion coefficients between III-V compounds and Si material are the major issues of the monolithic growth method, which will introduce a high density of threading dislocations (TDs) [4]. The APDs can be eliminated by using off-cut $\mathrm{Si}$ substrates and multi-step growth method. However, a tougher task is to deal with the high density of TDs. These TDs may propagate to the active region and cause non-radiative recombination, which leads to the degradation of laser devices. Strained layer superlattice (SLS) structures, i.e., dislocation filter layers (DFLs), could play a crucial role to suppress the propagations of TDs into the active region. Although the density of TDs could be reduced significantly after introducing DFLs, an advanced InAs/GaAs dot-in-a-well (DWELL) structure is desirable as the active region. This is because the unique properties of quantum dots (QDs), including low defect sensitivity, low threshold current density and temperature insensitive operation, 
which can lead to more stable and better performance of QD lasers compared with quantum well (QW) structure laser [14-16].

In this work, we first optimized the InGaAs/GaAs SLSs DFLs by adjusting growth conditions of GaAs spacer layer and structures of SLSs [17]. After that, based on this optimization of DFLs, we also combine the strategies of a nucleation layer made of AlAs and in-situ thermal annealing of DFLs to achieve a low dislocation density of $1 \times 10^{5} \mathrm{~cm}^{-2}$ in the III-V epilayers. These methods contribute to a high performance III-V QD on Si laser with a low threshold current of $62.5 \mathrm{~A} \mathrm{~cm}^{-2}$ and over $105 \mathrm{~mW}$ output power at room temperature under continuouswave (c.w.) operation. Finally, a lifetime study is implemented on this Si-based InAs/GaAs QD. Over 100,158 hours extrapolated lifetime suggest a high feasibility of delivering commercial III-V QD lasers directly grown on Si substrates [18].

\section{OPTIMIZATION OF DFLS OF INAS/GAAS QD LASERS ON SI}

\subsection{Epitaxial Growth structure}

In order to optimize the DFLs, a typical QD laser structure was grown on Si substrate by solid-source molecular beam epitaxy (MBE) as shown in Figure 1. A phosphorous-doped $\operatorname{Si}(100)$ wafer with a $4^{\circ}$ offcut to the [011] plane was used as the substrate. The Si substrate was first prepared by oxide desorption at $900{ }^{\circ} \mathrm{C}$ for $30 \mathrm{~min}$, and then a $1 \mu \mathrm{m} \mathrm{GaAs}$ buffer layer was grown with two steps: first $30 \mathrm{~nm}$ growth of GaAa layer at $380{ }^{\circ} \mathrm{C}$ with 0.1 monolayers/s (ML/s) growth rate and the remaining $970 \mathrm{~nm} \mathrm{GaAs}$ was grown at high temperature with 0.7 $\mathrm{ML} / \mathrm{s}$ growth rate. Three layers of InGaAs/GaAs SLSs for DFLs were utilized, each set of DFL including a 350 $\mathrm{nm}$ GaAs spacer layer and InGaAs/GaAs SLSs. In this DFL optimization, the first study is to investigate the growth condition of SLSs and GaAs spacer layer. Two different growth methods are introduced as illustrated in Fig. 2(a): in method I, a $350 \mathrm{~nm}$ GaAs spacer layer was grown with the temperature heating up from $420{ }^{\circ} \mathrm{C}$ to $610{ }^{\circ} \mathrm{C}$, while in method II, the GaAs layer was grown at a stable temperature of $610^{\circ} \mathrm{C}$. In both cases, InGaAs/GaAs SLSs were grown at $420^{\circ} \mathrm{C}$. In additional, we also studied the influence of indium composition and thickness of GaAs in $I n_{x} G a_{1-x} \mathrm{As} / \mathrm{GaAs}$ SLSs on the efficiency of DFLs by changing $x=0.16,0.18$ and 0.2 for indium composition and the GaAs thickness to 8, 9 and $10 \mathrm{~nm}$ in SLSs. For the active region of the laser structure, five sets of InAs/GaAs DWELL structure were sandwiched between GaAs/AlGaAs layers. Each set of DWELL was assembled by $3 \mathrm{ML}$ InAs QDs which were deposited on $2 \mathrm{~nm}$ InGaAs and cladded by $5 \mathrm{~nm}$ GaAs and $6 \mathrm{~nm}$ InGaAs. The DWELLs were grown at $510^{\circ} \mathrm{C}$ while each layer was separated by a $45 \mathrm{~nm}$ spacer layer of GaAs. Figure 2 (b) shows the AFM image of InAs/GaAs QDs with $3.8 \times 10^{10} \mathrm{~cm}^{-2}$ of dot density.

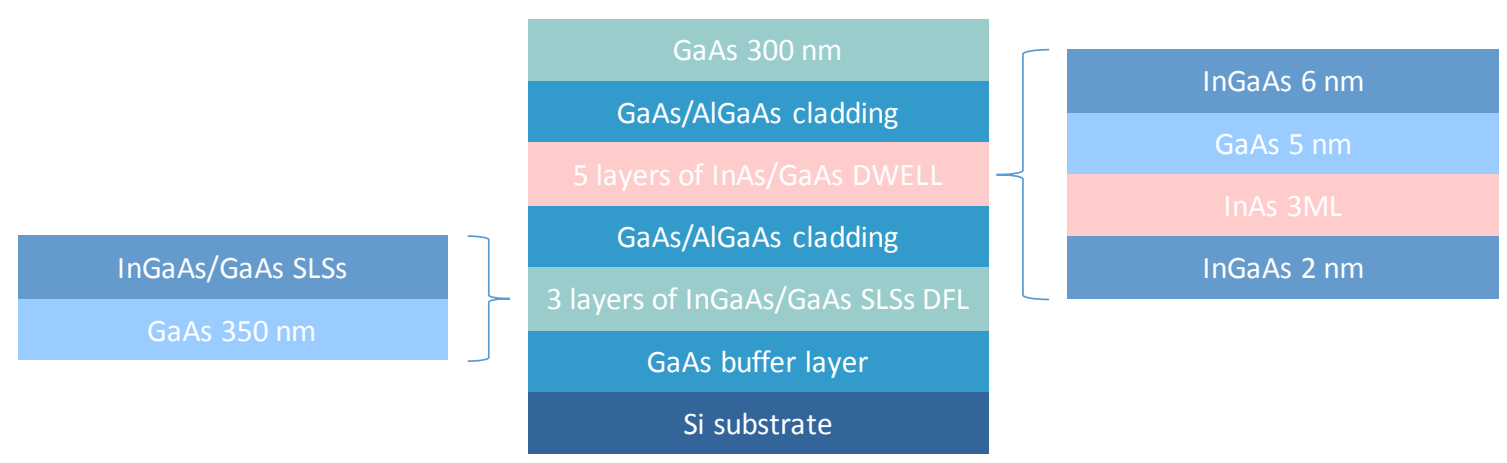

Figure 1. Schematic diagram of InAs/GaAs QD structures grown on a Si substrate. 



Figure 2. (a) The schematic of two growth method of DFLs. (b) AFM image of InAs/GaAs QDs.

\subsection{Results and Discussion}

To understand the effect of DFLs, photoluminescence (PL) peak intensity measurements are performed from sample A to F, which are shown in Table 1. These six samples are divided into three group studies: i) sample A and $\mathrm{B}$ used growth method I and II of spacer layers; ii) indium composition of $\operatorname{In}_{x} \mathrm{Ga}_{1-x} \mathrm{As}$ DFLs was changed to $x=0.16,0.18$ and 0.2 in sample C, B and D, respectively; iii) studies of the thickness of GaAs in SLSs were undertaken in samples B, E and F, which the thickness varies from $10 \mathrm{~nm}$ to $8 \mathrm{~nm}$. Fig. 3 gives the measurement results of PL peak intensity and full wave at half maximum (FWHM) of these three group studies at room temperature. When we used method II to grow GaAs spacer layer at temperature $610^{\circ} \mathrm{C}$, a three times stronger PL intensity was observed than that when the samples were grown by using method I (sample A), but the wavelength and FWHM keep almost unchanged as shown in Fig. 3(a). These PL emissions represent a significant improvement of DFLs using growth method II, and this advance can be attributed to the improved material quality after in-situ annealing of SLSs. Compared with growth method I, method II provides a high temperature growth condition for the GaAs spacer layer. This high temperature increases the motions of dislocations; this can lead them to meet and eliminate each other so that a low defect density was achieved on sample B. In this case, less non-radioactive recombination will occur in the active region, which could explain why a much higher PL peak intensity was achieved in growth method II.

In Fig.3 (b), the PL intensity spectra of samples B, C and D are presented with different indium composition of SLSs. When $x=0.18$, we achieved the maximum PL intensity value from sample B, which is about $30 \%$ and 40 $\%$ higher than that of sample C and D, respectively. Although the purpose of SLSs is to stop and annihilate dislocations, unfortunately a higher indium composition may cause an increase in strain-induced defects in DFLs instead of suppressing them. In contrast, a lower indium composition in InGaAs might lead to degrading the efficiency of DFLs in blocking the propagation of dislocations. In this study, a compromise of indium composition between the generation of strain-induced defects and the efficacy of SLSs was determined. It is a good balance of $x=0.18$, which provides the best crystal quantity.

Moreover, the effect of thickness of GaAs in SLSs on the performance of DFLs was investigated. As shown in Fig. 3(c), it is clear that the PL intensities of sample B and E have comparable values with $10 \mathrm{~nm}$ and $9 \mathrm{~nm}$ thickness of GaAs. However, the PL intensity of sample F, who has $8 \mathrm{~nm}$ thickness, is significant lower (50\%) than sample B and E. This could be explained by the accumulated strain in too thin GaAs layers in SLSs which degrades the quality of DFLs. To figure out the working details of DFLs, cross-sectional transmission electron microscopy (TEM) images of DFLs in sample B is shown in Fig. 4. As we can see, the three sets of SLSs can stop and annihilate TDs effectively. According to these three group studies, the most effective DFLs were grown by in-situ thermal annealing method with $x=0.18$ and $10 \mathrm{~nm}$ thickness of GaAs in SLSs. 
Table 1. The PL peak intensity and FWHM of samples with different growth method, indium composition and GaAs thickness.

\begin{tabular}{cccccc}
\hline Sample & Growth type & $\begin{array}{c}\mathbf{x} \text { of } \\
\ln _{\mathbf{x}} \mathrm{Ga}_{1-\mathrm{x}} \text { As }\end{array}$ & $\begin{array}{c}\text { GaAs } \\
\text { Thickness } \\
(\mathrm{nm})\end{array}$ & $\begin{array}{c}\text { PL peak } \\
\text { intensity (a.u) }\end{array}$ & FWHM (nm) \\
\hline A & I & 0.18 & 10 & 1.2 & 40.5 \\
B & II & 0.18 & 10 & 4 & 40.3 \\
C & II & 0.16 & 10 & 2.6 & 39.8 \\
D & II & 0.20 & 10 & 2.2 & 42 \\
E & II & 0.18 & 9 & 3.9 & 40.4 \\
F & II & 0.18 & 8 & 2 & 46.1 \\
\hline
\end{tabular}
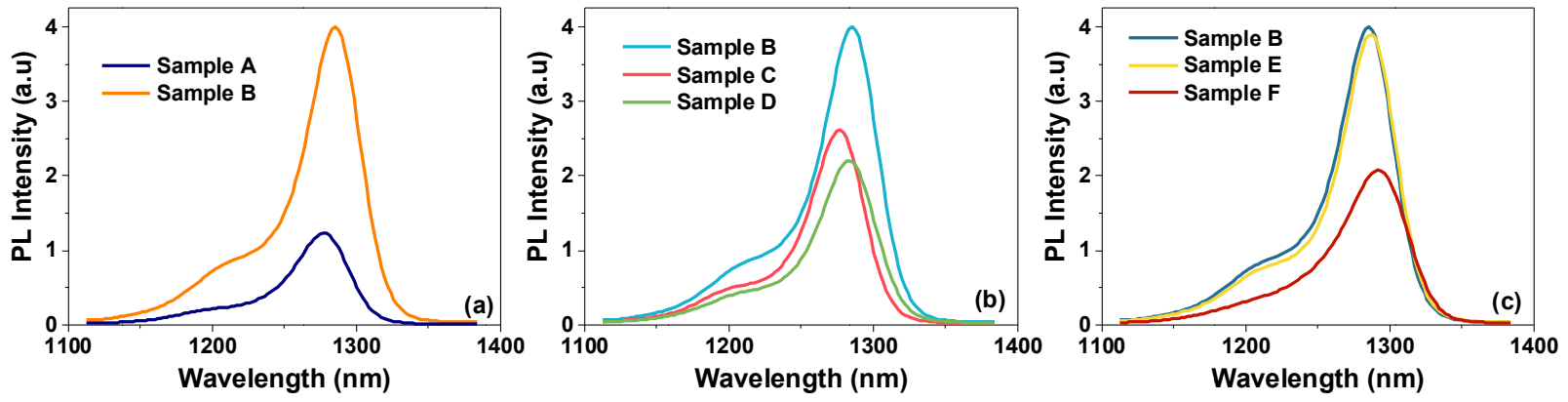

Fig.3 PL spectra of (a) sample A to B (b) sample B, C and D (c) sample B, E and F.

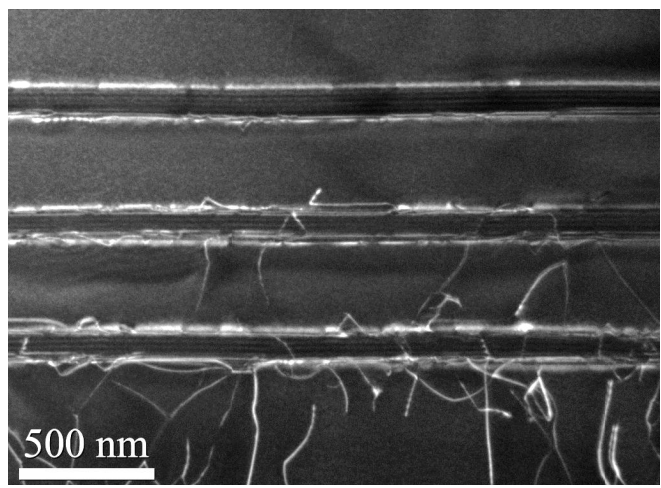

Fig 4. Bright-field TEM cross-section image of InGaAs/GaAs DFLs grow on a Si substrate. 


\section{LONG LIFE ELECTRICALLY PUMPED III-V QUANTUM DOTS LASER}

\subsection{Growth and fabrication of Laser structure}

Si-based QD laser devices have been further investigated by exploiting the results of optimization of DFLs in Section 2. A $4^{\circ}$ off-cut phosphorus-doped $\mathrm{Si}(100)$ substrate is used to annihilate APDs. In order to improve the epitaxy growth between the interface of Si and III-V compounds, an AlAs nucleation layer with $6 \mathrm{~nm}$ thickness (Fig. 5a) was grown first at a low growth temperature of $350{ }^{\circ} \mathrm{C}$. After that, a $1 \mu \mathrm{m}$ thick GaAs buffer layer was grown using a three-step growth technique at 350,450 and $590{ }^{\circ} \mathrm{C}$ for 30,170 and $800 \mathrm{~nm}$, respectively. Fig. 5 (b) is a TEM image of the GaAs buffer and DFLs. Most of dislocations are well confined in the first $200 \mathrm{~nm}$ buffer layer (position 1), which is attributed to the AlAs nucleation layer and good crystal of GaAs buffer layer using the three step growth method. Five sets of optimized $\mathrm{In}_{0.18} \mathrm{Ga}_{0.82} \mathrm{As} / \mathrm{GaAs}$ SLSs DFLs are grown with the purpose of reducing the high TD density and stopping propagation of TDs into the active region. In-situ thermal annealing of SLSs was also performed in the MBE reactor in order to further improve the efficacy of DFLs, by increasing the motion of defects and thereby raising the probability of annihilation between the defects. As shown in Fig.5 (c), the TD density before the first set of SLSs (position 1) is greater than $1 \times 10^{9} \mathrm{~cm}^{-2}$. It has been significantly reduced to the order of $1 \times 10^{5} \mathrm{~cm}^{-2}$ after five sets of SLSs. Five layers of InAs/GaAs DWELL structures with good QD uniformity and a dot density of around $3 \times 10^{10} \mathrm{~cm}^{-2}$ were formed as active region, where a PL emission at 1,300 nm was obtained (Fig. 6d). Each layer of DWELL comprised a 3ML InAs QD in the middle of $2 \mathrm{~nm} \mathrm{In}_{0.15} \mathrm{Ga}_{0.85}$ As and $6 \mathrm{~nm} \mathrm{In}_{0.15} \mathrm{Ga}_{0.85} \mathrm{As}$, and was separated by $50 \mathrm{~nm}$ GaAs spacer layer. The whole DWELL structures were sandwiched between $1.4 \mu \mathrm{m} \mathrm{Al}_{0.4} \mathrm{Ga}_{0.6} \mathrm{As}$-doped and $\mathrm{n}$-doped cladding layers. Finally, a $300 \mathrm{~nm}$ highly p-doped GaAs was grown as the contacting layer.

This lasers were fabricated as $50 \mu \mathrm{m}$ wide stripe broad-area lasers by using standard lithography and wet chemical etching techniques (Fig. 6). The ridge was etched to about $100 \mathrm{~nm}$ above the active region in order to give improved carrier confinement. Ti/Pt/Au and $\mathrm{Ni} / \mathrm{GeAu} / \mathrm{Ni} / \mathrm{Au}$ were deposited on the $\mathrm{p}^{+}-\mathrm{GaAs}$ contact layer and exposed $\mathrm{n}^{+}$- GaAs buffer layer to form the $\mathrm{p}$ - and $\mathrm{n}$ - contacts. The lasers were cleaved to mirror-like facets and no facet coating was applied, as shown in Fig. 6.
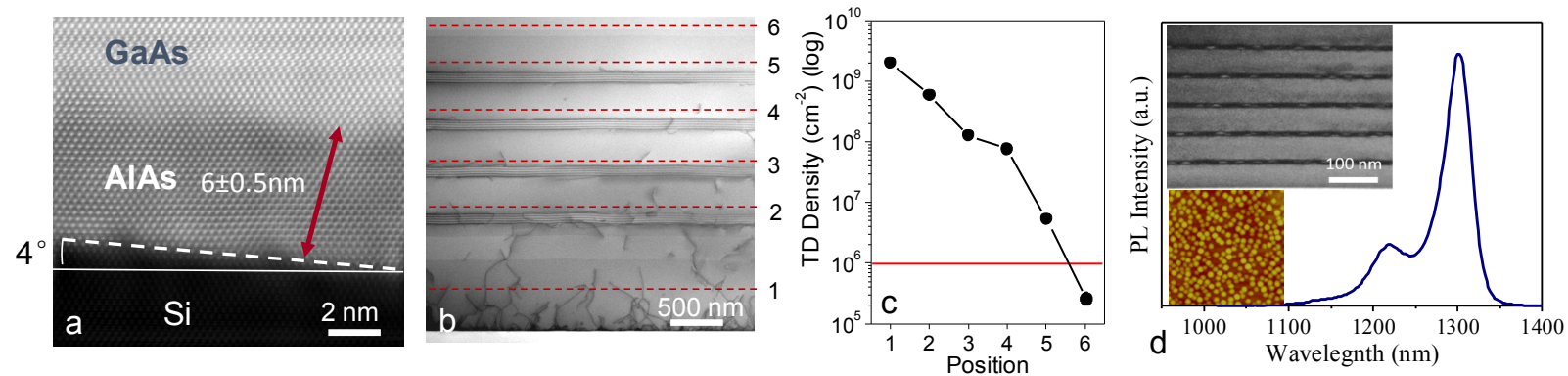

Figure 5. (a) $6 \mathrm{~nm}$ AlAs nuclear layer between $4^{\circ}$ offcut Si substrate and GaAs buffer layer (b) cross-section TEM image of DFLs with 6 positions (c) Dislocation density corresponding to 6 positions (d) PL spectrum of QD active region grown on Si. Inset: Bright-field TEM image of DWELL (top left), AFM image of uncapped QDs layer (bottom left)

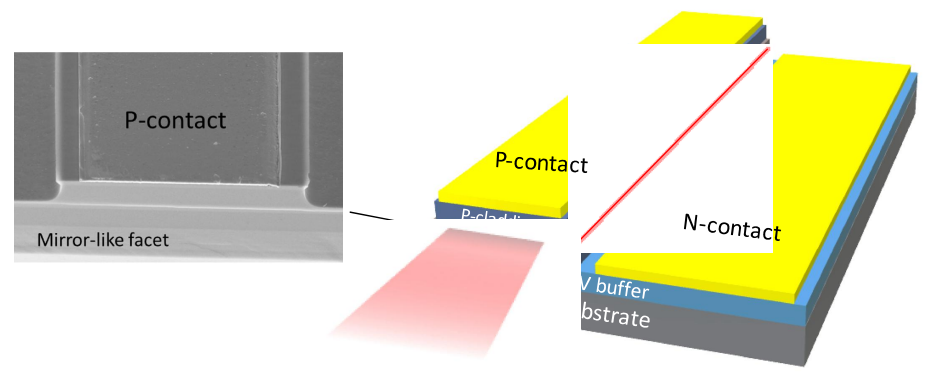

Figure 6. Schematic of InAs/GaAs QDs broad area Laser on Si substrate with mirror-like facet. 


\subsection{Results and Discussion}

Fig. 7 (a) shows the light-current-voltage (LIV) characteristic measurement of the III-V QD laser on Si under c.w. operation at room temperature. This laser achieved the extremely low threshold current density of $62.5 \mathrm{~A}$ $\mathrm{cm}^{-2}$ and over $100 \mathrm{~mW}$ output power when the injection current density is $650 \mathrm{~A} \mathrm{~cm}^{-2}$. Temperature dependence measurements were also carried out. The lasers can lase up to $75{ }^{\circ} \mathrm{C}$ heat-sink temperature in the c.w. mode (Fig.7 b) and up to $120{ }^{\circ} \mathrm{C}$ in pulsed mode. The characteristic temperature $\mathrm{T}_{0}$ has been calculated under pulsed operation as $51 \mathrm{~K}$ ranging from 20 to $60^{\circ} \mathrm{C}$ and $35 \mathrm{~K}$ from 70 to $120^{\circ} \mathrm{C}$. The temperature sensitivity can be improved by using p-doped QD and mounting the laser on a high thermal conductivity heat-sink in future work.

The emission spectra for this III-V QD on Si laser variation at different current densities were determined under c.w. operation at room temperature in Fig.7 (c). When the current density was $50 \mathrm{~A} \mathrm{~cm}^{-2}$, spontaneous emission was observed with broad FWHM of $38 \mathrm{~nm}$ at $1316 \mathrm{~nm}$ wavelength. As the injection current density was increased to $62.5 \mathrm{~A} \mathrm{~cm}^{-2}$, a typical lasing emission was observed with sharply increased intensity and narrowed FWHM $(2.4 \mathrm{~nm})$. At higher current densities, multi-mode lasing behaviour, characteristic of a broad area laser, was observed in the emission spectra. In addition, we implemented a lifetime study on this Si-based InAs/GaAs QD laser. The ageing test was undertaken at a constant c.w. drive current of $210 \mathrm{~mA}$ with the output power monitored, at a fixed temperature of $26^{\circ} \mathrm{C}$. The ageing results are depicted in Fig. $7 \mathrm{~d}$ where a $29.7 \%$ drop in power over the first $3,100 \mathrm{~h}$ continuous operation is observed. By fitting the threshold current trend with a sublinear model an extrapolated lifetime of over $100,158 \mathrm{~h}$ was determined.
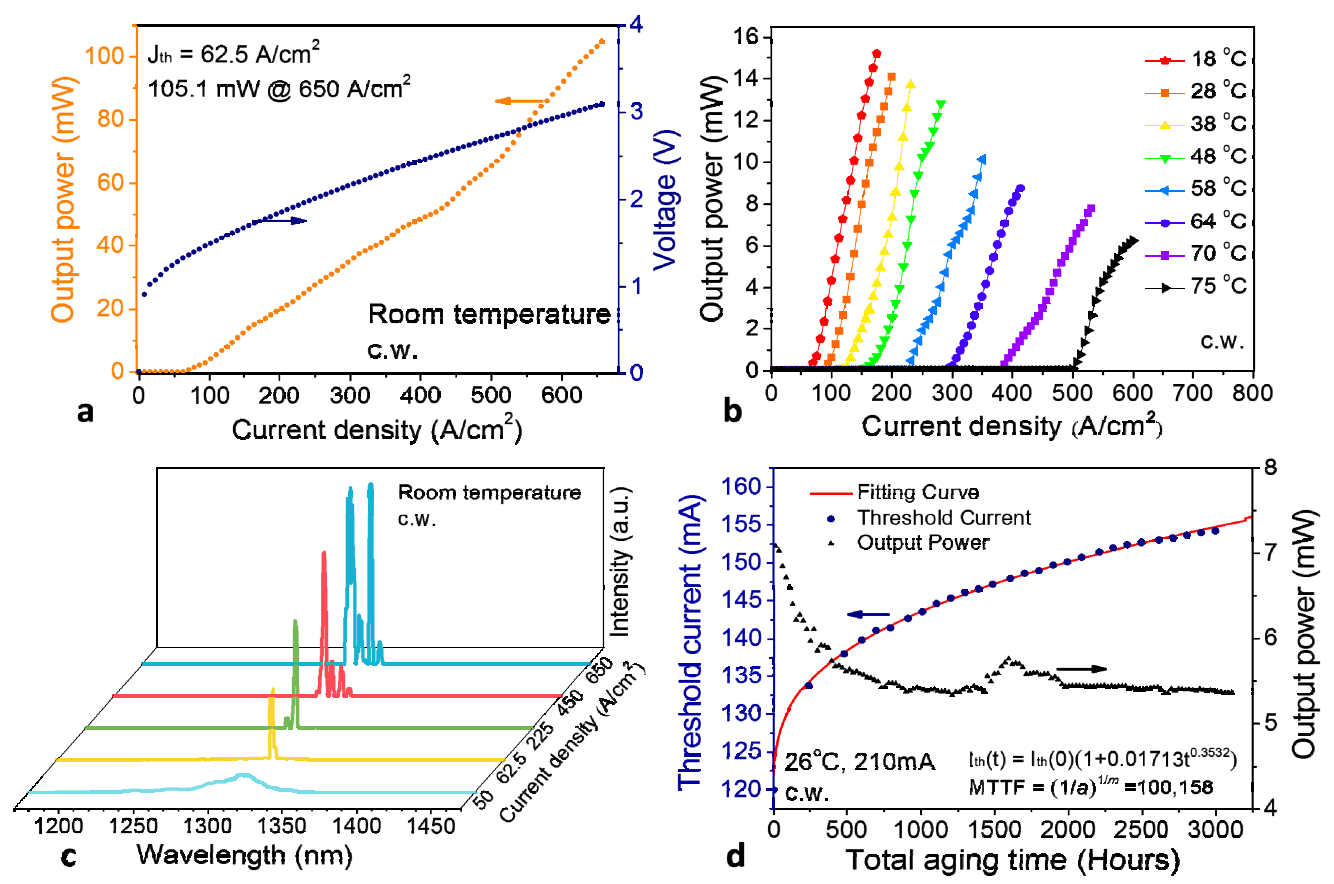

Figure 7. (a) Light-current-voltage (LIV) measurement of InAs/GaAs QDs laser on Si under c.w. operation at room temperature. (b) Light-current (L-I) characteristic of lasers at different temperatures under c.w. operation. (c) Emission spectra of Si-based lasers with different current densities under c.w. operstion. (d) Plots of threshold current and output power against total ageing time (hours) at $26^{\circ} \mathrm{C}$ with $210 \mathrm{~mA} \mathrm{c.w}$. drive current. 


\section{CONCLUSION}

In this paper, we have firstly investigated the optimization of InGaAs/GaAs SLSs DFLs with three variations: i) the growth condition of GaAs spacer layers; ii) the indium composition of InGaAs SLSs; and iii) the thickness of GaAs in SLSs. Comparison and analysis for each sample was carried out in order to achieve maximum efficacy of DFLs. This effort combined with the use of an AlAs nucleation layer, three step GaAs buffer layer growth and using QDs in the active region contributes to a remarkable result of an electrically-pumped III-V QD laser directly grown on Si substrate. Current density of $62.5 \mathrm{~A} \mathrm{~cm}^{-2}$ and over $105 \mathrm{mw}$ output power under c.w. operation were achieved from these lasers, which can lase up to $120^{\circ} \mathrm{C}$ under pulsed operation. Finally, an extrapolated lifetime of over 100,158 h lifetime provides a major step to delivering commercial III-V QD lasers on Si substrates.

\section{REFERENCE}

[1] Liang, D. and Bowers, J. E., "Recent progress in lasers on Silicon," Nat. Photonics 4(8), 511-517 (2010).

[2] G. T. Reed, G. Mashanovich, F. Y. Gardes, and D. J. Thomson, "Silicon optical modulators," Nat. Photonics 4(8), 518-526 (2010).

[3] Michel, J., Liu, J., Kimerling, L. C. High-performance Ge-on-Si photodetector. Nat. Photonics 4, 527-534 (2010).

[4] Chen, R., Tran, T.-T., Ng, K.W., Ko, W.S., Chang, L.C., Sedgwick, F. G. and Chang-Hasnain, C.: 'Nanolasers grown on silicon', Nat. Photonics 5(3), 170-175 (2011)

[5] Sugawara, M., Usami, M., "Quantum dot device handing the heat," Nat. Photonics, 3, 30-31 (2009).

[6] Liu, H., Wang, T., Jiang, Q., Hogg, R., Tutu, F., Pozzi, F. and Seeds, A., "Long-wavelength InAs/GaAs quantum-dot laser diode monolithically grown on Ge substrate," Nat. Photon., 5, 416419(2011).

[7] Wang, T., Liu, H., Lee, A., Pozzi, F., and Seeds, A., "1.3- $\mu \mathrm{m}$ InAs/GaAs quantum-dot lasers monolithically grown on Si substrates," Opt. Express 19(12), 11381-11386 (2011).

[8] Lee, A., Jiang, Q., Tang, M., Seed, A. and Liu, H., "Continuous-wave InAs/GaAs quantum-dot laser diodes monolithically grown on Si substrate with low threshold current densities," Opt. Express, 20, 22181-22187 (2012).

[9] Lee, A.D., Jiang, Q., Tang, M., Zhang, Y., Seeds, A.J. and Liu, H, "InAs/GaAs quantum-dot lasers monolithically grown on Si, Ge, and Ge-on-Si substrates," IEEE J. Sel. Top. Quantum Electron., 19, 1901107-1-1901107-7 (2013).

[10] Liu, A.Y., Zhang, C., Norman, J., Snyder, A., Lubyshev, D., Fastenau, J.M., Liu, A.W., Gossard, A.C. and Bowers, J.E., "High performance continuous wave $1.3 \mu \mathrm{m}$ quantum dot lasers on silicon," Appl. Phys. Lett., 104, 041104-1-041104-4 (2014).

[11]Liu, H., Childs, D., Badcock, T., Groom, K., Sellers, I., Hopkinson, M., Hogg, R., Robbins, D., Mowbray, D., and Skolnick, M., "High-performance three-layer 1.3- $\mu \mathrm{m}$ InAs-GaAs quantum-dot lasers with very low continuous-wave room-temperature threshold currents," IEEE Photon. Technol. Lett. 17, 1139-1141 (2005).

[12] Liu, A. Y., Herrick, R. W., Ueda, O., petroff, P. M., Gossard, A. C. and Bowers, J. E., "Reliability of InAs/GaAs Quantum Dot Lasers Epitaxially Grown on Silicon,” IEEE J. Select. Topics Quantum Electron, 21(6), 1900708 (2015).

[13] Chang. H. H. et al., "1310 nm silicon evanescent laser," Opt. Exp., vol. 15, no. 18, pp. 11466-11471 (2007).

[14] Tang, M., Chen, S., Wu, J., Jiang, Q., Dorogan, V.G., Benamara, M., Mazur, Y.I., Salamo, G.J., Seeds, A. and Liu, H., "1.3- $\mu \mathrm{m}$ InAs/GaAs quantum-dot lasers monolithically grown on Si substrates using InAlAs/GaAs dislocation filter layers," Opt. Express, 22, 11528-11535 (2014).

[15] Chen, S., Tang, M., Jiang, Q., Wu, J., Dorogan, V.G., Benamara, M., Mazur, Y.I., Salamo, G.J., Seeds, A. and Liu, H. "1.3- $\mu \mathrm{m}$ InAs/GaAs quantum-dot laser monolithically grown on Si Substrates operating over $100^{\circ} \mathrm{C}, "$ Electron. Lett., 50, 1467-1468 (2014). 
[16] Tang, M., Wu, J., Chen, S., Jiang Q., Seeds, A., Liu, H., Dorogan, V.G., Benamara, M., Mazur, Y.I., Salamo, G., "Optimisation of the dislocation filter layers in 1.3- $\mu \mathrm{m} \mathrm{InAs} / \mathrm{GaAs}$ quantum-dot lasers monolithically grown on Si substrates," J. IET Optoelectronics, 61-64 (2015).

[17] Tang, M., Chen, S., Wu, J., Kennedy, K., Jurczak, P., Liao, M., Beanland, R., Seeds, A. and Liu. H., "Optimizations of defect filter layers for 1.3- $\mu \mathrm{m}$ InAs-GaAs quantum-dot lasers monolithically grown on si substrates," IEEE JSTQE, vol. 22, no.6, 2551941(2016).

[18] Chen, S., Li, W., Wu, J., Jiang, Q., Tang, M., Shutts, S., Elliott, S.N., Sobiesierski, A., Seeds, A.J., Ross, I., Smowton, P.M., and Liu, H., "Electrically pumped continuous-wave III-V quantum dot lasers on silicon," Nature Photonics 10, 307-311 (2016) 